\title{
Manutenção da área foliar e produtividade de arroz irrigado com a aplicação de fertilizan- tes foliares no estádio de emborrachamento
}

\author{
Foliar area maintenance and yield with application of foliar fertilizers on booting stage of irrigated rice
}

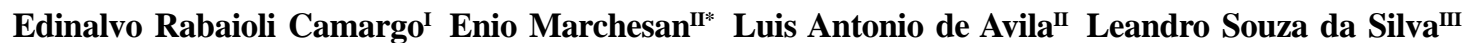 \\ Tiago Luis Rossato ${ }^{\mathrm{IV}}$ Paulo Fabrício Massoni ${ }^{\mathrm{IV}}$
}

\begin{abstract}
- NOTA -
RESUMO

A utilização de fertilizantes foliares, aplicados ao final do ciclo da cultura do arroz irrigado, pode proporcionar complementação nutricional para a planta e proteção contra patógenos com reflexos na produtividade. $O$ objetivo do estudo foi avaliar a utilização de fertilizantes foliares, aplicados no estádio de emborrachamento, sobre a produtividade do arroz irrigado. O experimento foi conduzido no ano agrícola 2005/ 2006, em área experimental de várzea do Departamento de Fitotecnia da Universidade Federal de Santa Maria. O

in a lowland area in Santa Maria-RS, Brazil. The treatments, arranged in a randomized block design with four replications, were constituted by 10 foliar fertilizers available in the market, two products applied to seeds, a fungicide, and a check treatment. The treatments presented no effects on any of the variables studied. The suitable climatic conditions and the fertilizer management carried out before the application of the foliar treatments led to the achievement of about 10,000 kg ha-1 yield, showing that there was no limitation on the level of yield obtained. The products utilized in this study did not increase the irrigated rice yield.
\end{abstract} delineamento experimental utilizado foi o de blocos ao acaso com quatro repetições. Os tratamentos foram compostos por 10 fertilizantes foliares, dois produtos aplicados via sementes, um fungicida e a testemunha. Os fertilizantes não influenciaram qualquer parâmetro avaliado. As condições climáticas e o manejo da adubação anterior à aplicação dos tratamentos foliares propiciaram condições favoráveis para o arroz expressar o seu potencial produtivo, em torno de $10.000 \mathrm{~kg} \mathrm{ha}^{-1}$. Neste nível de produtividade, os produtos utilizados não aumentaram a produtividade do arroz irrigado.

Palavras-chave: fosfito de potássio, micronutrientes, nitrogênio, Oryza sativa L., senescência foliar.

\section{ABSTRACT}

The use of foliar fertilizers applied to the reproductive phase of rice can improve the plant nutrition and protect the field against foliar diseases with gains in yield. The objective of this experiment was to evaluate the use of the foliar fertilizers applied to the booting stage in order to verify the irrigated rice yield. The experiment was carried out in 2005/06
Key words: potassium phosphate, micronutrients, nitrogen, Oryza sativa $L$., foliar senescence.

Na produção de arroz irrigado do Rio Grande do Sul, o aperfeiçoamento técnico e tecnológico, que vem sendo proposto pelos órgãos de pesquisa e adotado pelos produtores, tem propiciado incremento na produtividade. Para manutenção dessa condição ou mesmo superação dos patamares atuais, o manejo correto da nutrição de plantas torna-se indispensável. Nesse sentido, a utilização de fertilizantes foliares, aplicados próximos ao final do ciclo da cultura, poderá proporcionar uma complementação nutricional, no momento em que a translocação de fotoassimilados para os grãos é determinante para a produtividade (NTANOS \& KOUTROUBAS, 2002), atrasando a progressão da senescência foliar. O objetivo do presente estudo foi caracterizar a utilização de

\footnotetext{
IPrograma de Pós-graduação em Agronomia, Universidade Federal de Santa Maria (UFSM), Santa Maria, RS, Brasil.

IIDepartamento de Fitotecnia, Centro de Ciências Rurais (CCR), UFSM, 97105-900, Santa Maria, RS, Brasil. Email: emarch@ccr.ufsm.br.*Autor para correspondência.

IIIDepartamento de Solos, CCR, UFSM, Santa Maria, RS, Brasil.

${ }^{\text {IV }}$ Curso de Agronomia, UFSM, Santa Maria, RS, Brasil.
} 
fertilizantes foliares aplicados no estádio de emborrachamento na produtividade do arroz irrigado.

O experimento foi conduzido durante $\mathrm{o}$ ano agrícola de 2005/06, no Departamento de Fitotecnia da Universidade Federal de Santa Maria, em um solo classificado como Planossolo Hidromórfico eutrófico arênico, com as seguintes características físicoquímicas: argila $=34 \% ; \mathrm{pH}_{\text {agua }}(1: 1)=5,7 ; \mathrm{P}=9,3 \mathrm{mg} \mathrm{dm}^{-3}$; $\mathrm{K}=52 \mathrm{mg} \mathrm{dm}^{-3} ; \mathrm{Ca}=7,5 \mathrm{cmol} \mathrm{dm}^{-3} ; \mathrm{Mg}=3 \mathrm{cmol} \mathrm{dm}^{-3} \mathrm{e}$ M.O. $=2,5 \%$. O delineamento experimental utilizado foi de blocos ao acaso com quatro repetições. Os tratamentos foram compostos pela seleção de fertilizantes foliares disponíveis no mercado (T1 a T10), além de dois produtos aplicados via sementes (T11), um fungicida (T12) e uma testemunha (T13), que se encontram listados na tabela 1.

O preparo do solo para implantação da cultura foi realizado com gradagens e posterior aplainamento superficial do terreno, sendo a semeadura realizada em 03 de novembro de 2005, utilizando-se $110 \mathrm{~kg} \mathrm{ha}^{-1}$ de sementes da cultivar "IRGA 417”, tratada com inseticida. A adubação fosfatada e potássica foi realizada com a distribuição na linha de semeadura de $45 \mathrm{~kg} \mathrm{ha}^{-1}$ de $\mathrm{P}_{2} \mathrm{O}_{5}$ e $90 \mathrm{~kg} \mathrm{ha}^{-1}$ de $\mathrm{K}_{2} \mathrm{O}$. A emergência das plântulas ocorreu 12 dias após a semeadura, obtendose população média 200 plantas $\mathrm{m}^{-2}$ e o controle das plantas daninhas foi realizado aos 15 dias após a emergência (DAE). A primeira aplicação do fertilizante nitrogenado foi feita no estádio V4 do arroz (15 DAE), segundo escala de COUNCE et al. (2000), utilizando-se 90kg ha-1 de nitrogênio em solo não-inundado, seguida de irrigação definitiva da área. A segunda aplicação nitrogenada de cobertura foi realizada no estádio de iniciação da panícula (RO), aplicando-se $30 \mathrm{~kg} \mathrm{ha}^{-1} \mathrm{de}$ nitrogênio.

Os tratamentos foram aplicados durante o estádio de emborrachamento (R2), com exceção dos produtos Sol Seed + Zinco Sol 7 (T11), que foram aplicados como tratamento de semente. Os fertilizantes foliares foram aplicados no início do estádio R2 (74 DAE), já a aplicação do fungicida foi conduzida no final do estádio R2 (78 DAE), realizando a pulverização da mistura formulada de propiconazol + trifloxistrobina $\left(93,75+93,75 \mathrm{~g} \mathrm{ha}^{-1}\right)$. As aplicações foliares foram realizadas com pulverizador costal de precisão pressurizado a $\mathrm{CO}_{2}$, utilizando-se 200L ha-1 de calda. Os demais tratos culturais foram conduzidos conforme a recomendação técnica para a cultura.

O índice SPAD foi determinado por meio de um clorofilômetro, modelo SPAD 502 DL Meter (Minolta). A leitura média de cada tratamento foi resultante da observação de dez folhas bandeiras, realizando-se aferições nas extremidades e no centro de cada folha. Determinou-se ainda a senescência foliar por meio da observação visual das plantas na área da parcela. Para tanto, utilizou-se uma escala de 0 a 100 , que corresponde à porcentagem de folhas totalmente verdes e totalmente senescentes (cloróticas), respectivamente (CARLESSO et al., 1998).

Tabela 1 - Tratamentos aplicados na cultura do arroz irrigado, cultivar IRGA 417, com a respectiva dose e a quantidade aportada dos principais nutrientes presentes em cada produto. Santa Maria, RS. 2007.

\begin{tabular}{|c|c|c|c|}
\hline Trat. & Produto & Dose $\left(\mathrm{L} \mathrm{ha}^{-1}\right)$ & Quantidade aplicada (g ha $\left.{ }^{-1}\right)^{(3)}$ \\
\hline $\mathrm{T} 1$ & Fertamin Extra $^{\circledR}$ & 10,0 & $\mathrm{~N}(1240), \mathrm{S}(370), \mathrm{P}(120), \mathrm{K}(120)(4)$ \\
\hline $\mathrm{T} 2$ & Concorde $^{\circledR}$ & 2,0 & $\mathrm{~N}(230), \mathrm{P}(50), \mathrm{K}(26)(4)$ \\
\hline Т3 & Profol N $30^{\circledR}$ & 5,0 & $\mathrm{~N}(1960)$ \\
\hline $\mathrm{T} 4$ & Phosphorus $-\mathrm{k}^{\circledR} 00.28 .26$ & 3,0 & $\mathrm{P}(1209), \mathrm{K}(1123)$ \\
\hline $\mathrm{T} 5$ & Quimifol Arrank $^{\circledR}$ & 1,5 & Zn(95), S(76), Mn(57)(4) \\
\hline $\mathrm{T} 6$ & Quimifol Florada ${ }^{\circledR}+$ Quimióleo $^{\circledR}$ & $2,5+0,8$ & $\mathrm{Ca}(304), \mathrm{B}(34)$ \\
\hline $\mathrm{T} 7$ & Blooster $^{\circledR}$ & 1,0 & $\mathrm{Cu}(31), \mathrm{Mo}(21)(4)$ \\
\hline $\mathrm{T} 8$ & Supa-potássio ${ }^{\circledR}$ & 1,0 & $\mathrm{~K}(221), \mathrm{S}(78)$ \\
\hline T9 & Stimulate ${ }^{\circledR}+$ Natur'l óleo $^{\circledR}(1)$ & $0,25+1,0$ & citocinina $(0,022)$, ac. giberéli co $(0,012)$, auxina $(0,012)$ \\
\hline $\mathrm{T} 10$ & Crop Full XXI $I^{\circledR}+$ Pyto Sol PK ${ }^{\circledR}$ & $1,5+1,5$ & N (105), P(168), K(168), Ca(21), Zn(21)(4) + P(630), K(420) \\
\hline $\mathrm{T} 11$ & 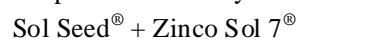 & $0,18+0,15$ & $\mathrm{~K}(24)$, Mo (10), $\mathrm{Zn}(2,4)(4)+\mathrm{Zn}(13)$ \\
\hline $\mathrm{T} 12$ & Stratego ${ }^{\circledR}(2)$ & 0,75 & (5) \\
\hline $\mathrm{T} 13$ & Testemunha & (5) & (5) \\
\hline
\end{tabular}

(1) Stimulate é um produto unicamente hormonal, possuindo em sua composição citocinina $\left(0,09 g \mathrm{~L}^{-1}\right)$, ácido giberélico $\left(0,05 g \mathrm{~L}^{-1}\right)$ e auxina $\left(0,05 \mathrm{~g} \mathrm{~L}^{-1}\right)$.

(2) Fungicida (mi stura comercial de propiconazol + trifloxistrobina).

(3) Quantidade calculada considerando a composição e a densidade de cada produto.

(4) Produto possui ainda outros nutrientes na composição, porém estes se encontram em quantidade inferior a 1\%, na unidade de peso por peso (p/p).

(5) Sem aplicação de nutriente via foliar e/ou via tratamento de semente.

Ciência Rural, v.38, n.5, ago, 2008. 
A avaliação da severidade de doenças foliares também foi realizada por meio de observações visuais, estimando-se a percentagem da área foliar atacada. A produtividade de grãos foi estimada em $6,0 \mathrm{~m}^{2}$ (5,0m x 1,2m), quando os grãos apresentavam umidade média de $22 \%$. Após a trilha, limpeza e pesagem dos grãos com casca, os dados foram corrigidos para $13 \%$ de umidade e convertidos para $\mathrm{kg} \mathrm{ha}^{-1}$. Os componentes da produtividade foram determinados pela contagem das panículas em um metro previamente demarcado e pela coleta de 10 panículas por ocasião da colheita. Os dados obtidos foram submetidos à análise de variância e as médias comparadas pelo teste de Tukey $(\mathrm{P}=0,05)$. Os dados de senescência foliar, severidade de doenças, esterilidade de espiguetas e rendimento industrial de grãos foram transformados para $y t=\sqrt{y+0,5}$.

Nas avaliações realizadas anteriormente à colheita, não foi verificado efeito dos tratamentos aplicados (Tabela 2). A porcentagem de folhas senescentes aumentou no período de enchimento de grãos, sendo que os valores médios situavam-se em 5\% no estádio R6 (elongação de pelo menos uma cariopse até a extremidade da casca) e atingiram $27 \%$ em R8 (formação de grãos com casca marrom). Este é um comportamento natural durante o período de enchimento de grãos, decorrente da impossibilidade de formação de folhas novas e da necessidade de remobilização de nitrogênio, que aceleram a senescência foliar e resultam no decréscimo da atividade fotossintética (OOKAWA et al., 2003). A severidade de mancha parda (Drechslera oryzae), única doença foliar verificada na área, foi consideravelmente baixa nas duas avaliações realizadas. Nesta condição, até mesmo o tratamento fúngico (T12) não propiciou efeito diferenciado para o controle das doenças foliares. Assim, a observação de possível efeito protetor dos tratamentos compostos por fosfitos de potássio, tais como o Phosphorus-k (T4) e Pyto Sol PK (T10) foi impossibilitada.

Não houve diferença entre os tratamentos para o índice de SPAD nas avaliações realizadas durante o período de enchimento de grãos, mesmo para produtos contendo nitrogênio, havendo um decréscimo de 35 para 24 entre a avaliação em R5 (85 DAE) e R8 (115 DAE). A redução do índice SPAD relaciona-se com o aumento da senescência foliar, ocasionando o declínio da capacidade fotossintética, que é decorrente de alterações funcionais da unidade fotossintética (HIDEMA, 1991).

A produtividade de grãos e os seus componentes não foram afetados pelos tratamentos (Tabela 3). As condições climáticas e o manejo

Tabela 2 - Senescência e severidade de doenças foliares aos 90 (R6) e 105 (R8) dias após a emergência (DAE) e índice SPAD na folha bandeira aos 85 (R5), 100 (R7) e 115 (R8) DAE, em resposta à aplicação dos tratamentos na cultura do arroz imigado, cultivar IRGA 417. Santa Maria, RS. 2007.

\begin{tabular}{|c|c|c|c|c|c|c|c|}
\hline \multirow{2}{*}{ Tratamento(1) } & \multicolumn{2}{|c|}{-----Senescência (\%)----- } & \multicolumn{2}{|c|}{---Se veridade (\%)---- } & \multicolumn{3}{|c|}{-----------Índice SPAD----------- } \\
\hline & R6(2) & $\mathrm{R} 8(2)$ & R6 & R8 & $\mathrm{R} 5(2)$ & $\mathrm{R} 7(2)$ & R8 \\
\hline $\mathrm{T} 1$ & $5 n s$ & $25 n s$ & Ons & $0,6 \mathrm{~ns}$ & $37 n s$ & $32 n s$ & $25 n s$ \\
\hline $\mathrm{T} 2$ & 5 & 30 & 0 & 0,5 & 36 & 33 & 24 \\
\hline T3 & 5 & 21 & 0 & 0,6 & 37 & 34 & 27 \\
\hline $\mathrm{T} 4$ & 6 & 28 & 0 & 0,9 & 36 & 32 & 24 \\
\hline $\mathrm{T} 5$ & 5 & 21 & 0 & 0,5 & 36 & 33 & 28 \\
\hline T6 & 5 & 26 & 0 & 0,4 & 37 & 34 & 24 \\
\hline $\mathrm{T} 7$ & 5 & 30 & 0 & 0,5 & 36 & 30 & 20 \\
\hline $\mathrm{T} 8$ & 5 & 28 & 0 & 0,5 & 37 & 31 & 23 \\
\hline T9 & 4 & 30 & 0 & 0,8 & 37 & 31 & 22 \\
\hline T10 & 5 & 26 & 0,1 & 0,6 & 37 & 32 & 20 \\
\hline T11 & 5 & 28 & 0 & 0,6 & 37 & 33 & 26 \\
\hline $\mathrm{T} 12$ & 6 & 34 & 0 & 0,8 & 35 & 31 & 20 \\
\hline $\mathrm{T} 13$ & 6 & 24 & 0 & 0,6 & 36 & 32 & 23 \\
\hline Média & 5 & 27 & 0 & 0,6 & 36 & 32 & 24 \\
\hline CV (\%) & 12 & 13 & 6 & 14 & 4 & 7 & 19 \\
\hline
\end{tabular}

(1) [T1] Fertamin Extra ${ }^{\circledR}$; [T2] Concorde ${ }^{\circledR}$; [T3] Profol N 30 ${ }^{\circledR}$; [T4] Phosphorus-k ${ }^{\circledR} 00.28 .26$; [T5] Quimifol Arrank ${ }^{\circledR}$; [T6] Quimifol Florada ${ }^{\circledR}$ + Quimióleo ${ }^{\circledR}$; [T7] Blooster ${ }^{\circledR}$; [T8] Supa-potássio ${ }^{\circledR}$; [T9] Stimulate ${ }^{\circledR}+$ Natur’óleo $^{\circledR}$; [T10] Crop Full XXI ${ }^{\circledR}+$ Pyto Sol PK $^{\circledR}$; [T11] Sol Seed ${ }^{\circledR}$ + Zinco Sol ${ }^{\circledR}$ 7; [T12] Stratego ${ }^{\circledR}$; [T13] Testemunha. Doses e quantidades aplicadas dos principais nutrientes presentes em cada produto na tabela 1.

(2) Estádio de desenvolvimento segundo escala proposta por COUNCE et al. (2000).

ns Teste F não-significativo $(\mathrm{P}=0,05)$.

Ciência Rural, v.38, n.5, ago, 2008. 
Tabela 3 - Produtividade, panículas por metro quadrado (PMQ), número de grãos por panícula (GP), massa de mil grãos (MMG), esterilidade de espiguetas (EE) e rendimento de engenho (RE), em resposta à aplicação dos tratamentos na cultura do arroz irrigado, cultivar "IRGA 417”. Santa Maria, RS. 2007.

\begin{tabular}{|c|c|c|c|c|c|c|}
\hline Tratamento(1) & Produtividade (kg ha ${ }^{-1}$ ) & PMQ & GP & MMG (g) & EE (\%) & RE (\%) \\
\hline $\mathrm{T} 1$ & $9.634 \mathrm{~ns}$ & 521ns & 91ns & $24,8 \mathrm{~ns}$ & $4,9 \mathrm{~ns}$ & $62 \mathrm{~ns}$ \\
\hline $\mathrm{T} 2$ & 10.442 & 504 & 97 & 24,6 & 3,8 & 63 \\
\hline T3 & 10.073 & 462 & 83 & 25,6 & 3,9 & 64 \\
\hline $\mathrm{T} 4$ & 10.019 & 537 & 89 & 25,1 & 5,2 & 62 \\
\hline T5 & 9.797 & 462 & 90 & 24,4 & 4,0 & 62 \\
\hline T6 & 10.025 & 529 & 99 & 23,9 & 4,1 & 63 \\
\hline $\mathrm{T} 7$ & 9.577 & 459 & 96 & 24,3 & 4,9 & 63 \\
\hline $\mathrm{T} 8$ & 10.313 & 546 & 101 & 23,8 & 5,3 & 62 \\
\hline T9 & 9.791 & 466 & 98 & 24,9 & 3,8 & 64 \\
\hline $\mathrm{T} 10$ & 9.738 & 496 & 82 & 25,7 & 4,0 & 63 \\
\hline $\mathrm{T} 11$ & 9.928 & 549 & 88 & 23,9 & 5,7 & 62 \\
\hline $\mathrm{T} 12$ & 10.597 & 491 & 98 & 24,8 & 3,8 & 64 \\
\hline $\mathrm{T} 13$ & 10.387 & 497 & 98 & 24,4 & 4,9 & 63 \\
\hline Média & 10.025 & 501 & 93 & 24,6 & 4,5 & 63 \\
\hline CV (\%) & 5 & 12 & 13 & 4 & 8 & 2 \\
\hline
\end{tabular}

(1) [T1] Fertamin Extra ${ }^{\circledR}$; [T2] Concorde ${ }^{\circledR}$; [T3] Profol N 30 ${ }^{\circledR}$; [T4] Phosphorus-k ${ }^{\circledR} 00.28 .26$; [T5] Quimifol Arrank ${ }^{\circledR}$; [T6] Quimifol Florada ${ }^{\circledR}$ + Quimióleo ${ }^{\circledR}$; [T7] Blooster ${ }^{\circledR}$; [T8] Supa-potássio ${ }^{\circledR}$; [T9] Stimulate ${ }^{\circledR}+$ Natur'óleo $^{\circledR}$; [T10] Crop Full XXI ${ }^{\circledR}+$ Pyto Sol PK $^{\circledR}$; [T11] Sol Seed ${ }^{\circledR}$ + Zinco Sol $^{\circledR}$ 7; [T12] Stratego ${ }^{\circledR}$; [T13] Testemunha. Doses e quantidades aplicadas dos principais nutrientes presentes em cada produto na Tabela 1.

ns Teste F não-significativo $(\mathrm{P}=0,05)$.

nutricional conduzido anteriormente à aplicação dos tratamentos foram suficientes para obtenção de produtividade superior a $10.000 \mathrm{~kg} \mathrm{ha}^{-1}$, indicando que não houve limitação para os níveis produtivos obtidos. Com relação aos tratamentos contendo micronutrientes (T5, T6, T7 e T11), resultados semelhantes foram obtidos em estudo conduzido durante três anos por MARCHESAN et al., (2001) em que não se verificou efeito na produtividade. Nos níveis de produtividade obtidos, a utilização de fertilizantes foliares na fase reprodutiva não promoveu acréscimo na produtividade e na qualidade física dos grãos de arroz irrigado.

\section{REFERÊNCIAS}

CARLESSO, R. et al. Índice de área foliar e altura de plantas de arroz submetidas a diferentes práticas de manejo. Revista Brasileira de Engenharia Agrícola e Ambiental, v.2-3, p.268-272, 1998.
COUNCE, P.A. et al. A uniform, objective and adaptive system for expressing rice development. Crop Science, n.40, p.436443, 2000.

HIDEMA, J. et al. Photosynthetic characteristics of rice leaves under different irradiances from full expansion through senescence. Plant Physiology, v.97, p.1287-1293, 1991.

MARCHESAN, E. et al. Adubação foliar com micronutrientes em arroz irrigado, em área sistematizada. Ciência Rural, v.31, n.6, p.941-945, 2001.

NTANOS, D.A.; KOUTROUBAS, S.D. Dry matter and N accumulation and translocation for Indica and Japonica rice under Mediterranean conditions. Field Crops Research, v.74, p.93-101, 2002.

OOKAWA, T. et al. A comparasion of the accumulation and partitioning of nitrogen in plants between two rice cultivares, Akenohoshi and Nipponbare, at the ripening stage. Plant Production Science, v.6, n.3, p.172-178, 2003. 